\title{
Poly(ADP-ribose) polymerase inhibitor olaparib hampers placental growth factor-driven activation of myelomonocytic cells
}

\author{
PEDRO MIGUEL LACAL ${ }^{1}$, MARIA GRAZIA ATZORI ${ }^{2}$, FEDERICA RUFFINI ${ }^{1}$, \\ LUCIO TENTORI $^{2}$ and GRAZIA GRAZIANI ${ }^{2}$ \\ ${ }^{1}$ Laboratory of Molecular Oncology, 'Istituto Dermopatico dell'Immacolata'-IRCCS, I-00167 Rome; \\ ${ }^{2}$ Department of Systems Medicine, University of Rome 'Tor Vergata', I-00133 Rome, Italy
}

Received August 29, 2017; Accepted February 15, 2018

DOI: $10.3892 /$ or.2018.6291

\begin{abstract}
The vascular endothelial growth factor receptor-1 (VEGFR-1) is a tyrosine kinase receptor activated by the angiogenic factors VEGF-A and placental growth factor (PIGF). While VEGF-A binds to both VEGFR-1 and VEGFR-2, PIGF interacts exclusively with VEGFR-1 triggering a signaling pathway involved in: i) tumor-associated angiogenesis; ii) chemotaxis and invasion of the extracellular matrix (ECM) by cancer cells; and iii) mobilization of bone marrow-derived myeloid cells that generate tumor-associated macrophages. By using a novel anti-VEGFR-1 monoclonal antibody (D16F7 $\mathrm{mAb}$ ), which hampers receptor activation without avoiding ligand binding, we recently demonstrated that VEGFR-1 blockade reduced myeloid progenitor mobilization and monocyte/macrophage cell infiltration of tumor grafts in vivo. Since poly(ADP-ribose) polymerase (PARP)-1 exerts a pro-inflammatory role favoring monocyte activation, in the present study we investigated whether the PARP inhibitor (PARPi) olaparib hampers PIGF-induced activation of human myelomonocytic cells. HL-60 cells induced to differentiate towards the monocytic/macrophage lineage were tested and the results were confirmed in freshly isolated monocytes obtained from
\end{abstract}

Correspondence to: Professor Grazia Graziani, Department of Systems Medicine, University of Rome 'Tor Vergata', Via Montpellier 1, I-00133 Rome, Italy

E-mail: graziani@uniroma2.it

Dr Pedro Miguel Lacal, Laboratory of Molecular Oncology, 'Istituto Dermopatico dell'Immacolata'-IRCCS, Via dei Monti di Creta 104, I-00167 Rome, Italy

E-mail: p.lacal@idi.it

Abbreviations: PIGF, placental growth factor; VEGFR, vascular endothelial growth factor receptor; PARP, poly(ADP-ribose) polymerase; PARPi, PARP inhibitor

Key words: PARP inhibitor, PlGF, VEGFR-1, myelomonocytic cells, cell migration, melanoma healthy donors. Cells were treated with olaparib, at clinically achievable concentrations, before exposure to PlGF and were analyzed for migration and ECM invasion in response to PIGF. Olaparib effects were compared to those obtained with D16F7 $\mathrm{mAb}$ used as single agent or in combination with the PARPi. The results indicate that differentiated HL-60 cells and monocytes expressed VEGFR-1 and migrated in response to PlGF. Moreover, olaparib and D16F7 inhibited PIGF-induced chemotaxis and ECM invasion in a dose-dependent manner and with similar efficacy. However, in combination studies the PARPi and D16F7 did not exert synergistic effects. Olaparib also hampered PlGF-induced monocyte adhesion to fibronectin, while it did not affect $\mathrm{NF}-\kappa \mathrm{B}$ activation in response to the angiogenic factor. These data suggest that olaparib likely interferes with the same pathway affected by the anti-VEGFR-1 $\mathrm{mAb}$ and that inhibition of PlGF-induced monocyte activation may contribute to PARPi antitumor activity.

\section{Introduction}

The placental growth factor (PlGF) is a member of the vascular endothelial growth factor (VEGF) family of angiogenic factors that shares with VEGF-A the ability to activate the transmembrane tyrosine kinase receptor type 1 (VEGFR-1). However, differently from VEGF-A, which also interacts with VEGFR-2, PIGF binds exclusively to VEGFR-1. Besides being expressed in endothelial cells during vessel formation and remodeling, VEGFR-1 is present in monocytes/macrophages and in a variety of human cancers, where it favors cell migration and survival predicting poor prognosis and recurrence $(1,2)$. Notably, in myelomonocytic cells the PIGF/VEGFR-1 pathway plays an important role in cell recruitment within the tumor mass, tumor-associated angiogenesis and metastasis $(3,4)$. Moreover, VEGFR-1 contributes to the differentiation of $\mathrm{CD}_{11} \mathrm{~b}^{+}$myelomonocytic cells from hematopoietic progenitors (5) and identifies a monocyte subset with increased chemotactic response towards VEGF-A and PIGF (6). VEGFR-1 activation by PlGF is also involved in the downregulation of type $1 \mathrm{~T}$ helper immune responses by modulating the function of dendritic cells (7) and macrophage polarization toward a tumor-associated macrophage (TAM) 
subtype that releases matrix metalloproteinase-9 (MMP-9) (8). Stimulation of monocytes by PlGF through activation of VEGFR-1 results in triggering of PI3 kinase/Akt and ERK-1/2 pathways and gene expression induction with increased production of cytokines (TNF- $\alpha$ and IL-1b) or chemokines (MCP-1, IL-8 and MIP-1b) (9).

In addition to enhancing tumor aggressiveness, PlGF and VEGFR-1 overexpression may also contribute to primary or acquired tumor resistance to current anti-VEGF-A therapies. Indeed, we observed increased PIGF plasma levels in melanoma patients treated with the anti-VEGF-A monoclonal antibody (mAb) bevacizumab compared to healthy donors (10). One of the mechanisms by which activation of the PIGF/VEGFR-1 pathway may result in failure of anti-VEGF therapies relies on stimulation of myelomonocytic cell recruitment to the tumor mass $(3,11)$. We recently demonstrated that in vivo treatment of melanoma-bearing mice with the anti-VEGFR-1 D16F7 $\mathrm{mAb}$ strongly inhibited mobilization of myeloid progenitor cells from the bone marrow and drastically reduced monocyte/macrophage infiltration at the tumor border in melanoma nodules (11). The anti-VEGFR-1 mAb also exerted inhibitory effects on migration and/or extracellular matrix (ECM) invasion by endothelial cells as well as cancer cells (melanoma and glioblastoma) in response to VEGF-A and PIGF (11-13). The D16F7 $\mathrm{mAb}$ has a novel mechanism of action since it hampers VEGFR-1 activation without preventing ligand binding.

Poly(ADP-ribose) polymerase (PARP)-1 is the most abundant isoform of an enzyme family capable of synthesizing ADP-ribose polymers (PAR) that are transferred to PARP-1 itself and to a number of target proteins. Thus, PARylation represents a transient post-translational modification of proteins that is involved in the regulation of various cellular functions, including DNA repair and maintenance of genomic integrity, gene transcription and cell death (reviewed in refs. 14,15). Moreover, PARP-1 plays an important role in inflammation, either because it acts as a transcriptional regulator capable of modulating the expression of pro-inflammatory genes or because, when overactivated, it leads to $\mathrm{NAD}^{+}$and ATP depletion with consequent necrosis that initiates the inflammatory process (reviewed in ref. 14). In this regard, PARP inhibition exerts protective effects blocking the pro-inflammatory activity of PARP-1. Indeed, treatment with PARP inhibitors (PARPi) of macrophages diminished the production of inflammatory mediators (16) and decreased monocytes adhesion and migration across the blood-brain barrier (BBB) in in vitro models by reducing the activation of specific integrins (17).

PARPi have been largely investigated for cancer treatment in combination with chemo- or radiotherapy and as monotherapy in the case of tumors deficient in homologous recombination DNA repair (18). Among the multiple PARPi in clinical development, olaparib, rucaparib and niraparib have been recently approved. In particular, olaparib is the first orally bioavailable agent to receive approval by both FDA and EMA as maintenance monotherapy of patients with platinum-sensitive relapsed BRCA-mutated ovarian cancer and by FDA for BRCA-mutated/HER2-negative metastatic breast cancer $(19,20)$. Moreover, it is currently being studied in a number of clinical trials for a variety of solid tumors (www.ClinicalTrials.gov). We recently demonstrated that olaparib exerts cytotoxic effects against acute myeloid leukemia blasts, while it does not affect the viability of bone marrow $\mathrm{CD} 34^{+}$enriched peripheral blood cells obtained from healthy donors (21).

In the present study, we analyzed the effect of the PARPi olaparib on activation of human myelomonocytic cells by PIGF and found that olaparib and D16F7 similarly inhibited PlGF-induced chemotaxis and ECM invasion in a dose-dependent manner. Results demonstrate that inhibition of monocyte activation mediated by PIGF may contribute to the antitumor activity of PARPi. Moreover, these data are expected to be relevant for designing new therapeutic strategies for neoplastic and inflammatory disorders where PIGF has been demonstrated to play an important role.

\section{Materials and methods}

Cell cultures, human monocyte isolation and drug treatment. The human promyelocytic HL-60 cell line was obtained from the American Type Culture Collection (ATCC; Manassas, VA, USA). Cells were cultured in RPMI-1640 medium (Sigma-Aldrich, St. Louis, MO, USA) supplemented with $10 \%$ fetal bovine serum (FBS; Sigma-Aldrich), $2 \mathrm{mM}$ L-glutamine, $100 \mathrm{U} / \mathrm{ml}$ penicillin and $100 \mu \mathrm{g} / \mathrm{ml}$ streptomycin sulfate, at $37^{\circ} \mathrm{C}$ in a $5 \% \mathrm{CO}_{2}$ humidified atmosphere. HL-60 cells were authenticated by STR profiling (BMR Genomics, Padova, Italy).

For differentiation towards monocytic/macrophagic cells, the HL-60 cell line was treated with $10 \mathrm{ng} / \mathrm{ml}$ phorbol 12-myristate 13-acetate (PMA; Sigma-Aldrich) for $24 \mathrm{~h}$.

Peripheral blood mononuclear cells were separated by Ficoll-Hypaque density centrifugation of buffy coats obtained from adult healthy donors. Monocytes were isolated from peripheral blood mononuclear cells by plastic adherence for $2 \mathrm{~h}$ at $37^{\circ} \mathrm{C}$ in a $5 \% \mathrm{CO}_{2}$ humidified atmosphere. After removal of non-adherent cells by repeated washing with serum-free Dulbecco's modified Eagle's medium (DMEM; Sigma-Aldrich), adherent monocytes were collected by gentle scraping with a plastic scraper.

For the treatment of differentiated HL-60 cells or monocytes with olaparib, cells were exposed to the indicated concentrations of the PARPi for $2 \mathrm{~h}$ at $37^{\circ} \mathrm{C}$. The stock solution of olaparib (40 mM; Selleckchem, Munich, Germany), was prepared by dissolving the drug in dimethyl sulfoxide (DMSO; Sigma-Aldrich). Control cells were always exposed to DMSO at a concentration equal to that of the drug-treated cells. For the analysis of the influence of olaparib on maximally stimulated PARP activity, differentiated HL-60 cells were incubated with $50 \mu \mathrm{M} \mathrm{H}_{2} \mathrm{O}_{2}$ for 15 min after treatment with the PARPi.

D16F7 stock solution was prepared in phosphate-buffered saline (PBS) and in vitro treatment was performed by incubating the cells in a rotating wheel for $30 \mathrm{~min}$ at room temperature. The generation of the anti-VEGFR-1 D16F7 $\mathrm{mAb}$ was previously described (11).

Analysis of VEGFR-1 transcript. Quantification of the membrane VEGFR-1 transcript was performed by real-time quantitative reverse transcription-polymerase chain reaction (qRT-PCR) according to the dual-labeled fluorigenic probe method and using an ABI Prism 7000 sequence detector 
(PerkinElmer, Groningen, The Netherlands), as previously described (22). Expression levels were calculated by the relative standard curve method. Primers used were as follows: VEGFR-1, forward 5'-ACCGAATGCCACCTCCATG-3' and reverse 5'-AGGCCTTGGGTTTGCTGTC-3'. For each sample, the level of VEGFR-1 transcript was normalized to that of $18 \mathrm{~S}$ RNA (TaqMan ${ }^{\circledR}$ Gene Expression Assay; Applied Biosystems, Foster City, CA, USA) and compared to the VEGFR-1-negative M14 melanoma cell line, to which the arbitrary value of 1 was assigned.

Western blot analysis. Proteins were run using $10 \%$ SDS-polyacrylamide gels and transferred to supported nitrocellulose membranes by standard techniques. Immunodetection was performed using the following primary antibodies: Anti-PAR mouse mAb (1:1,000; cat. no. 4335-MC-100; Trevigen, Gaithersburg, MD, USA), rabbit anti-E-cadherin and anti- $\beta$-catenin mAbs (1:1,000; cat. no. 3195P and $8480 \mathrm{P}$, respectively; Cell Signaling Technology, Danvers, MA, USA) or rabbit polyclonal anti- $\beta$-actin (1:10,000; cat. no. A2066; Sigma Aldrich) antibodies. Anti-mouse or anti-rabbit IgG/horseradish peroxidase secondary antibodies (1:1,000; cat. no. 170-6516 and 170-6515, respectively; Bio-Rad Laboratories, Hercules, CA, USA) and ECL Western Blotting detection reagents from GE Healthcare (cat. no. RPN2106; Milan, Italy), were used to identify the proteins of interest.

Chemotaxis and ECM invasion assays. In vitro migration assay was performed using Boyden chambers equipped with $8-\mu \mathrm{m}$ pore diameter polycarbonate filters (Nuclepore; Whatman Inc., Clifton, NJ, USA) (for differentiated HL-60 cells), as previously described (23), or Corning HTS Transwell ${ }^{\circledR}-96$ permeable support plates (Sigma-Aldrich) with 5.0- $\mu \mathrm{m}$ pore polycarbonate membranes (for monocytes), coated with $5 \mu \mathrm{g} / \mathrm{ml}$ gelatin (Sigma-Aldrich). Vehicle, olaparib and/or D16F7 pre-treated cells were loaded in the upper compartment of Boyden chambers $\left(2 \times 10^{5} \mathrm{HL}-60\right.$ cells/chamber $)$ or Transwell plates $\left(1.5 \times 10^{5}\right.$ monocytes/chamber). Migration assay, toward serum-free medium [containing $0.1 \%$ bovine serum albumin (BSA) and $1 \mu \mathrm{g} / \mathrm{ml}$ heparin] or serum-free medium containing PlGF (50 $\mathrm{ng} / \mathrm{ml}$ ), present in the lower compartment, was performed in the absence or in the presence of olaparib and/or D16F7 mAb at the concentrations and incubation times specified in the Figure legends. Migrated cells, attached to the lower side of the filters, were fixed in ethanol, stained with crystal violet and counted in triplicate samples for a total of 12 high power microscopic fields (x200 and x400 magnification for HL-60 cells and monocytes, respectively).

Invasion assay with differentiated HL-60 cells was performed in Boyden chambers $\left(2 \times 10^{5}\right.$ cells/chamber $)$ equipped with $8-\mu \mathrm{m}$ pore diameter polycarbonate filters coated with $20 \mu \mathrm{g}$ of the commercial basement membrane matrix Matrigel (BD Biosciences, Buccinasco, Italy), as previously described (11).

Cell survival assay. Cell culture viability was analyzed using the tetrazolium compound MTS [3-(4,5-dimethylthiazol2-yl)-5-(3-carboxymethoxyphenyl) 2-(4-sulphophenyl)-2Htetrazolium, inner salt] from Promega (Madison, WI, USA).
Increasing numbers of differentiated HL-60 cells, untreated or pretreated with olaparib for $2 \mathrm{~h}$, were seeded in sextuplicate into flat-bottom 96 -well plates and cultured at $37^{\circ} \mathrm{C}$ in a $5 \%$ $\mathrm{CO}_{2}$ humidified atmosphere. After $24 \mathrm{~h}, 20 \mu \mathrm{l}$ of $2 \mathrm{mg} / \mathrm{ml}$ MTS solution was added to each well and cells were incubated at $37^{\circ} \mathrm{C}$ for $2 \mathrm{~h}$. Absorbance was read at $490 \mathrm{~nm}$ (reference wavelength $655 \mathrm{~nm}$ ) using a 3550-UV Microplate reader (Bio-Rad Laboratories).

Analysis of $N F-\kappa B$ activity. NF- $\kappa \mathrm{B}$ activity was determined utilizing a NF- $\kappa \mathrm{B}$ p65 ELISA-based transcription factor assay kit (TransAM assay; Active Motif Europe, Rixensart, Belgium). The assay was performed according to the manufacturer's protocol using $20 \mu \mathrm{g}$ of whole-cell extracts. The kit contains a 96-well plate with immobilized oligonucleotides comprising a NF- $\kappa$ B consensus site (5'-GGGACTTTCC-3') to which the p65 active form specifically binds. The NF- $\kappa \mathrm{B}$ detecting antibody recognizes an epitope on p65 that is accessible only when this protein is activated and bound to its target DNA. After incubation with a horseradish peroxidase-conjugated secondary antibody, NF- $\kappa \mathrm{B}$ activity was quantified by a microplate reader 3550-UV (Bio-Rad Laboratories) at $450 \mathrm{~nm}$ with a reference wavelength of $655 \mathrm{~nm}$.

Cell adhesion to fibronectin. Cell adhesion was tested by seeding monocytes $\left(1.5 \times 10^{5}\right.$ cells/well) into flat-bottom 96-well plates previously coated with fibronectin (5 $\mu \mathrm{g} / \mathrm{m}$; Sigma-Aldrich) and blocked with $1 \%$ BSA/PBS. Selected wells were coated with $1 \% \mathrm{BSA} / \mathrm{PBS}$ only to evaluate background cell adhesion. After $2 \mathrm{~h}$ of incubation at $37^{\circ} \mathrm{C}$, non-adherent cells were washed out and attached cells were fixed in ethanol and stained with crystal violet. Adhesion efficiency was determined by counting, in quadruplicate samples, the number of adherent cells/microscopic field for a total of 12 high power fields (x200 magnification).

Statistical analyses. For multiple comparisons ANOVA followed by Bonferroni's post-test was used. Statistical significance was determined at $\alpha=0.05$ level. Differences were considered statistically significant when $\mathrm{P}<0.05$.

\section{Results}

The PARPi olaparib inhibits migration and ECM invasion triggered by PlGF in HL-60 cells differentiated to monocyte/macrophage-like cells. As a model of myelomonocytic cells, we initially used the HL-60 cell line induced to differentiate towards the monocytic/macrophage lineage by treatment with PMA. As indicated by the results of qRT-PCR analysis (Fig. 1A), differentiation of HL-60 cells was accompanied by the induction of high VEGFR-1 transcript levels and the presence of this receptor rendered cells responsive to PlGF, as previously demonstrated (11). In order to select the concentration of olaparib capable of abrogating maximally stimulated PARP activity in this model, differentiated HL-60 cells were pretreated with graded concentrations of the PARPi $\left(0.1,1\right.$ and $2 \mu \mathrm{M}$, for $2 \mathrm{~h}$ ), exposed to $\mathrm{H}_{2} \mathrm{O}_{2}$ for $15 \mathrm{~min}$ and then analyzed by western blotting to detect PARylated proteins. In fact, the oxidant $\mathrm{H}_{2} \mathrm{O}_{2}$ is known to generate DNA strand breaks and to induce PARP-1 overactivation. Results indicated 
A

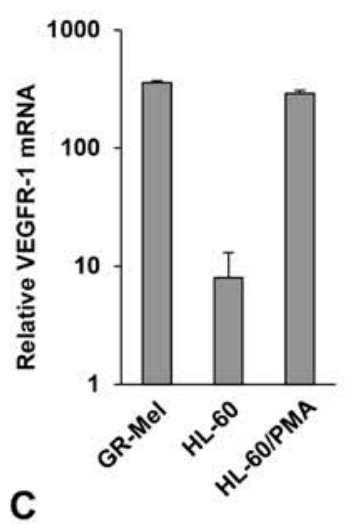

B
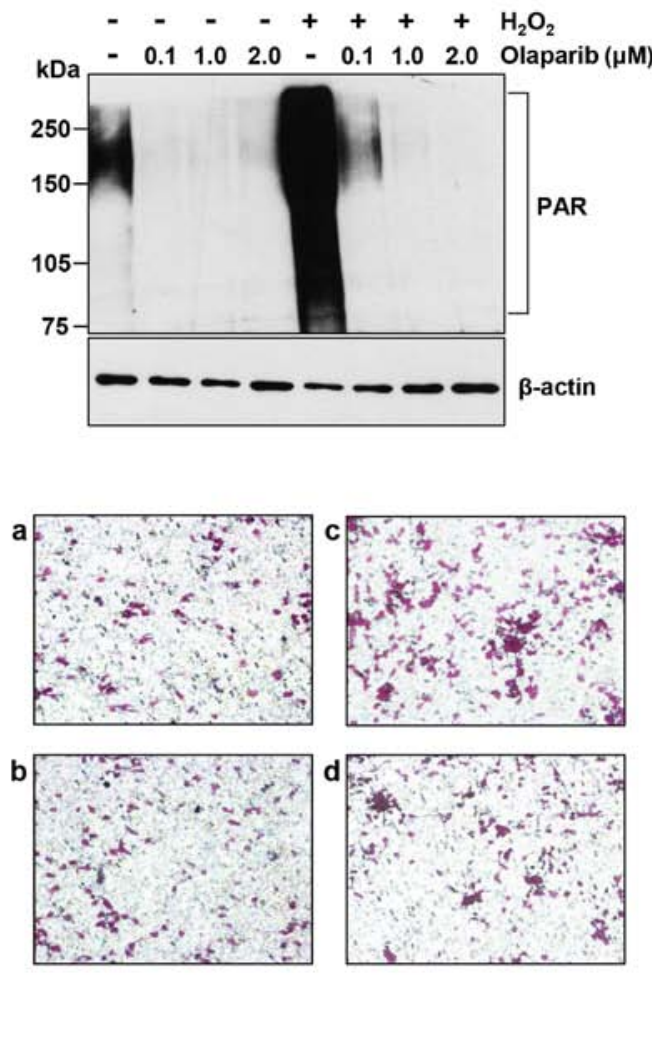
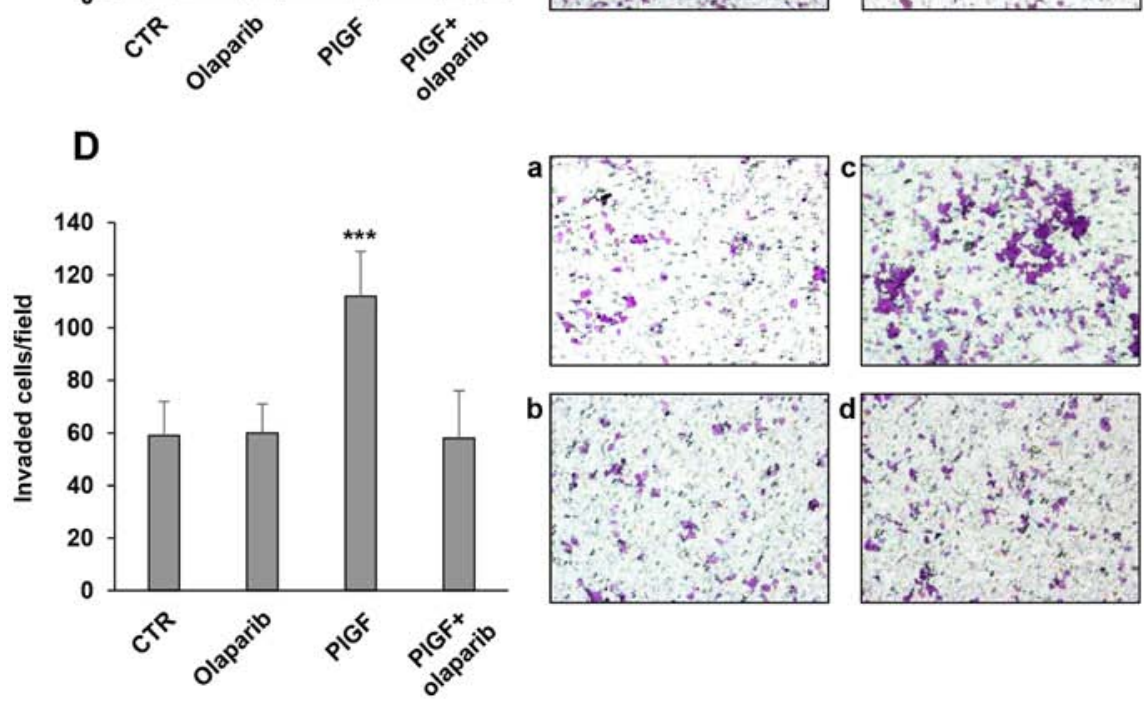

Figure 1. Influence of the PARPi olaparib on ECM invasion induced by PIGF in HL-60 cells differentiated in vitro to the monocyte/macrophage lineage. (A) Analysis of VEGFR-1 expression. Total RNA, extracted from undifferentiated or PMA-differentiated HL-60 cells, was analyzed by qRT-PCR analysis. The human melanoma GR-Mel cells were used as positive control and the results are in relation to the VEGFR-1-negative M14 melanoma cell line, to which the arbitrary value of 1 was assigned. (B) Inhibitory effect of olaparib on protein PARylation in differentiated HL-60 cells. The analysis of PAR-modified proteins was performed by western blotting in cells exposed to $50 \mu \mathrm{M} \mathrm{H}_{2} \mathrm{O}_{2}$ for 15 min after treatment with vehicle or olaparib (at the indicated $\mu \mathrm{M}$ concentrations for $2 \mathrm{~h}$ ). (C) Influence of olaparib on differentiated HL-60 cell migration induced by PIGF. Migration of differentiated HL-60 cells, vehicle or olaparib $(2 \mu \mathrm{M}$ for $2 \mathrm{~h}$ ) pre-treated, in response to serum-free medium (CTR and olaparib) or $50 \mathrm{ng} / \mathrm{ml} \mathrm{PlGF}$ was evaluated in Boyden chambers (4-h incubation) equipped with gelatin-coated filters. Histogram represents the mean values of the number of migrated cells/field \pm SD of three independent determinations. Results of statistical analysis using one-way ANOVA followed by Bonferroni's post-test were as follows: ${ }^{* * *} \mathrm{P}<0.001$, PlGF vs. CTR, PIGF vs. olaparib, PlGF vs. PlGF +olaparib; differences between CTR, olaparib and PlGF + olaparib were not significant. Representative images of differentiated HL-60 cell migration are shown (magnification, x100): a, untreated non-stimulated control cells (CTR); b, olaparib-treated non-stimulated cells; c, PlGF-stimulated cells; d, PlGF+olaparib. (D) Influence of olaparib on ECM invasion induced by PIGF. Invasion of differentiated HL-60 cells, vehicle or olaparib $(2 \mu \mathrm{M}$ for $2 \mathrm{~h})$ pre-treated, in response to serum-free medium (CTR and olaparib) or $50 \mathrm{ng} / \mathrm{ml} \mathrm{PlGF}$ was evaluated in Boyden chambers (4-h incubation) equipped with Matrigel-coated filters. Histogram represents the mean values of the number of invaded cells/field \pm SD of three independent determinations. Results of statistical analysis using one-way ANOVA followed by Bonferroni's post-test were as follows: ${ }^{* * *} \mathrm{P}<0.001$, PIGF vs. CTR, PlGF vs. olaparib, PlGF vs. PlGF +olaparib; differences between CTR, olaparib and PIGF + olaparib were not significant. Representative images of differentiated HL-60 cell invasion are shown (magnification, x100): a, untreated non-stimulated control cells (CTR); b, olaparib-treated non-stimulated cells; c, PlGF-stimulated cells; d, PIGF+olaparib.

that at a concentration as low as $0.1 \mu \mathrm{M}$, olaparib markedly inhibited basal or $\mathrm{H}_{2} \mathrm{O}_{2}$-induced PARP-1 activation and consequent protein PARylation, which was totally abrogated by 1 and $2 \mu \mathrm{M}$ olaparib (Fig. 1B). Notably, exposure of HL-60 
A
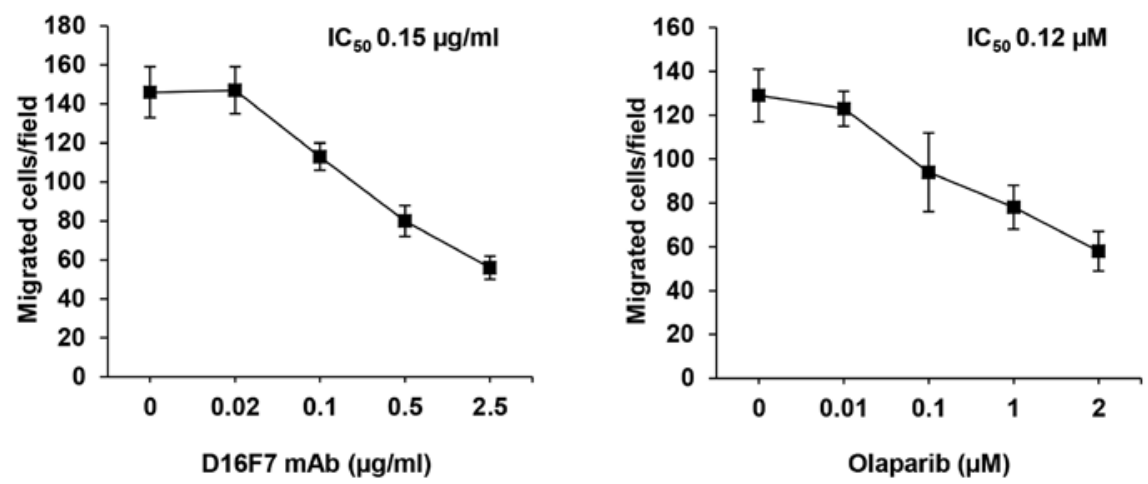

B

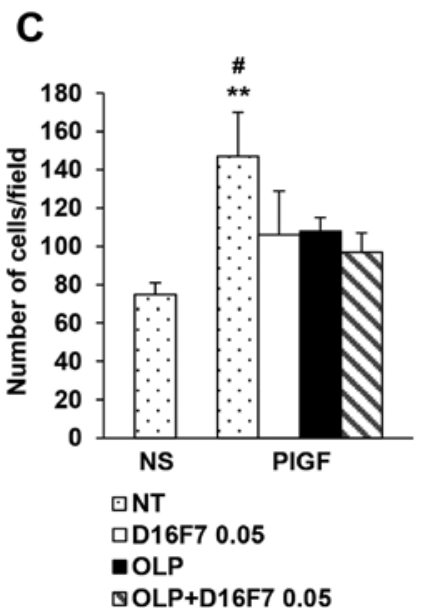

Figure 2. Analysis of the influence of olaparib, as a single agent or in combination with the anti-VEGFR-1 D16F7 mAb, on PlGF-induced chemotaxis and ECM invasion of differentiated HL-60 cells. (A) Dose-dependent inhibitory effect of D16F7 mAb or olaparib on PlGF-induced chemotaxis. Migration of differentiated HL-60 cells, vehicle ( 0 in the x-axis) and D16F7 mAb or olaparib pre-treated (at the indicated concentrations), for $30 \mathrm{~min}$ or $2 \mathrm{~h}$, respectively, in response to PlGF (50 ng/ml) was evaluated in Boyden chambers equipped with gelatin-coated filters. (B) Influence of olaparib and D16F7 mAb combined treatment on differentiated HL-60 cell migration in response to PlGF. Migration assay was performed using vehicle (not treated, NT) or D16F7 pre-treated cells in the absence or in the presence of a fixed concentration of olaparib (OLP, $0.1 \mu \mathrm{M})$. Results of statistical analysis using one-way ANOVA followed by Bonferroni's post-test were as follows: ${ }^{* * *} \mathrm{P}<0.001$, PlGF vs. all groups (except PlGF $+0.05 \mu \mathrm{g} / \mathrm{ml} \mathrm{D} 16 \mathrm{~F} 7$ ). Differences between PlGF $+0.1 \mu \mathrm{g} / \mathrm{ml} \mathrm{D} 16 \mathrm{~F} 7$ and PlGF $+0.1 \mu \mathrm{g} / \mathrm{ml}$ D16F7 + olaparib and differences between PlGF $+0.2 \mu \mathrm{g} / \mathrm{ml}$ D16F7 and PlGF $+0.2 \mu \mathrm{g} / \mathrm{ml}$ D16F7 +olaparib were not significant. NS, non-stimulated cells. (C) Influence of olaparib and D16F7 mAb combined treatment on ECM invasion by differentiated HL-60 cells in response to PlGF. Invasion assay was performed using vehicle (not treated, NT) or D16F7 pre-treated cells $\left(0.05 \mu \mathrm{g} / \mathrm{ml}\right.$, i.e., $\mathrm{mAb} \mathrm{IC}_{50}$ on ECM invasion) in the absence or in the presence of a fixed concentration of olaparib (OLP, $0.1 \mu \mathrm{M}$ ). Results of statistical analysis using one-way ANOVA followed by Bonferroni's post-test were as follows: ${ }^{* *} \mathrm{P}<0.01, \mathrm{PlGF}$ vs. non-stimulated cells (NS); ${ }^{\prime} \mathrm{P}<0.05$, PLGF vs. all the other groups. Differences between PlGF $+0.05 \mu \mathrm{g} / \mathrm{ml} \mathrm{D} 16 \mathrm{~F} 7, \mathrm{PlGF}+\mathrm{olaparib}$ and $\mathrm{PlGF}+0.05 \mu \mathrm{g} / \mathrm{ml}$ D16F7 + olaparib were not significant. Histograms represent the mean values of the number of migrated cells/field $\pm \mathrm{SD}$ of three independent determinations.

differentiated cells to olaparib $(2 \mu \mathrm{M})$ markedly inhibited migration (Fig. 1C) and ECM invasion triggered by PlGF to background values (Fig. 1D). At this concentration, olaparib did not significantly affect the viability of differentiated HL-60 cells (data not shown).

Olaparib inhibits PlGF-induced response similarly to the anti-VEGFR-1 D16F7 $m A b$. In order to evaluate whether olaparib treatment might synergize with VEGFR-1 blockade, cells were treated with olaparib, D16F7 or both agents and then exposed to PIGF. Treatment with the anti-VEGFR-1 D16F7 $\mathrm{mAb}$, which is known to hamper PIGF-induced VEGFR-1 activation (11), markedly affected migration of PMA-differentiated HL-60 cells with an $\mathrm{IC}_{50}$ value of $0.15 \pm 0.06 \mu \mathrm{g} / \mathrm{ml}$ (Fig. 2A). Olaparib inhibited cell migration in a dose-dependent manner (Fig. 2A) with an $\mathrm{IC}_{50}$ of $0.12 \pm 0.01 \mu \mathrm{M}$. Cells were also treated with olaparib at its $\mathrm{IC}_{50}$ value in combination with

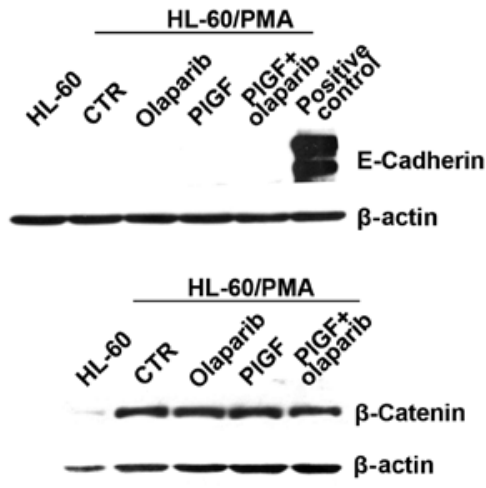

Figure 3. Analysis of the influence of olaparib treatment on the expression of EMT markers. The analysis of E-cadherin and $\beta$-catenin expression was performed by western blotting in cells pretreated with $2 \mu \mathrm{M}$ olaparib for $2 \mathrm{~h}$ before exposure to $50 \mathrm{ng} / \mathrm{ml}$ PlGF for $20 \mathrm{~h}$. $\beta$-actin was used as loading control. Protein extracts from the human epidermoid carcinoma A431 cell line were used as a positive control for the analysis of E-cadherin. 
A

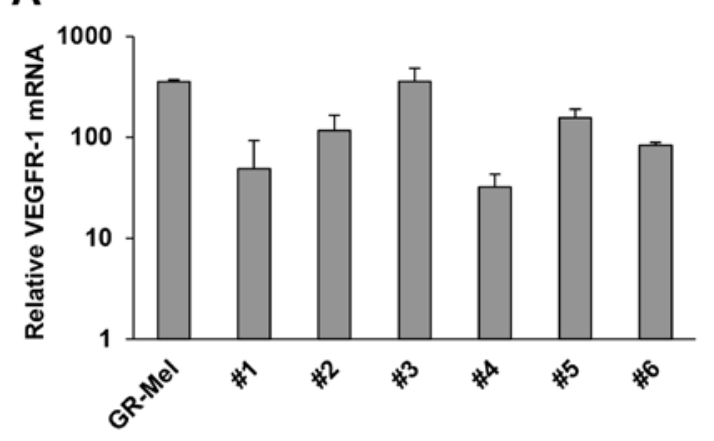

C
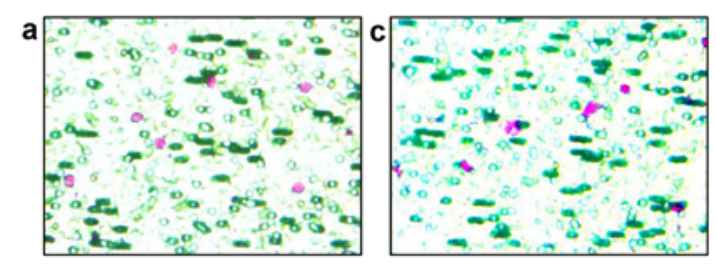

b)

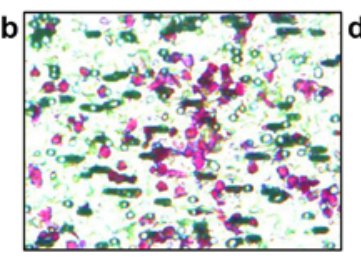

B

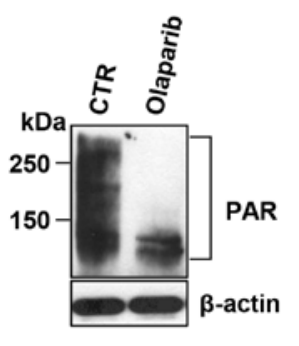

D

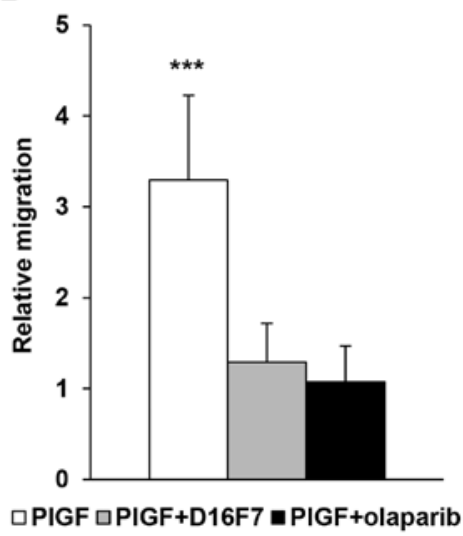

Figure 4. Inhibitory activity of olaparib and the anti-VEGFR-1 D16F7 mAb on the chemotactic response of human monocytes to P1GF. (A) Analysis of VEGFR-1 expression. Total RNA was extracted from freshly isolated human monocytes obtained from 6 healthy donors and analyzed by qRT-PCR. The human melanoma GR-Mel cells were used as positive control and results were in relation to the VEGFR-1-negative M14 melanoma cell line, to which the arbitrary value of 1 was assigned. (B) Inhibitory effect of olaparib on protein PARylation in human monocytes. The analysis of PAR-modified proteins was performed by western blotting in vehicle or olaparib pre-treated ( $2 \mu \mathrm{M}$ for $2 \mathrm{~h}$ ) monocytes. (C) Influence of olaparib and D16F7 mAb, as single agents or in combination, on PlGF-induced monocyte chemotaxis. Monocytes were exposed to $2 \mu \mathrm{M}$ olaparib for $2 \mathrm{~h}$ and/or to $5 \mu \mathrm{g} / \mathrm{ml} \mathrm{D} 16 \mathrm{~F} 7 \mathrm{for} 30 \mathrm{~min}$ and cell migration was analyzed in Corning HTS Transwell ${ }^{\circledR}-96$ permeable support plates equipped with gelatin-coated filters (1.5x10 ${ }^{5}$ cells/well, 2-h incubation). Representative images of monocyte migration are shown (magnification, $\mathrm{x} 400$ ); a, untreated non-stimulated control; b, PlGF stimulated cells; c, PlGF + D16F7 $\mathrm{mAb} ; \mathrm{d}, \mathrm{PlGF}+$ olaparib. (D) Results of monocyte chemotaxis analysis are expressed as the ratio between the number of migrated monocytes stimulated with PIGF and that of non-stimulated, untreated control cells. Data are the mean values obtained from 6 different monocyte preparations. Results of statistical analysis using one-way ANOVA followed by Bonferroni's post-test were as follows: ${ }^{* * *} \mathrm{P}<0.001, \mathrm{PlGF}$ vs. PlGF $+\mathrm{D} 16 \mathrm{~F} 7$ and $\mathrm{PlGF}$ vs. PlGF + olaparib; differences between PlGF+D16F7 and PlGF+olaparib were not significant.

D16F7 at concentrations encompassing values above and below the $\mathrm{mAb} \mathrm{IC}_{50}(0.05,0.1$ and $0.2 \mu \mathrm{g} / \mathrm{ml})$. Results indicated that the PARPi and D16F7 did not exert synergistic effects (Fig. 2B), suggesting that olaparib might interfere with the same pathway affected by the anti-VEGFR-1 mAb. Similar results were obtained by testing ECM invasion induced by PIGF (Fig. 2C). D16F7 was more effective in inhibiting cell invasiveness as compared to chemotaxis $\left(\mathrm{mAb} \mathrm{IC}_{50}: 0.05 \pm 0.01 \mu \mathrm{g} / \mathrm{ml}\right)$. When olaparib and $\mathrm{D} 16 \mathrm{~F} 7$ were combined at their $\mathrm{IC}_{50}$ values, the inhibitory effect on ECM invasion was similar to that obtained with the single agents (Fig. 2C).

In order to evaluate whether the inhibitory effect of olaparib on PIGF-induced ECM invasion was due to modulation of epithelial to mesenchymal transition (EMT) markers, we tested the expression of E-cadherin and $\beta$-catenin in differentiated HL-60 cells exposed to PIGF and olaparib by western blot analysis. The results showed that cells did not express E-cadherin, while they expressed high levels of $\beta$-catenin, which is compatible with a mesenchymal phenotype. However, no modulation of protein expression was observed in response to the different treatments (Fig. 3).
Olaparib inhibits PARP activity and PlGF-induced chemotaxis of freshly isolated human monocytes. Primary human monocytes were isolated from buffy coats obtained from 6 healthy donors and analyzed for the presence of the VEGFR-1 transcript. Results of qRT-PCR indicated that VEGFR-1 was detected in all monocyte samples tested even though at different levels (Fig. 4A). Analysis by western blotting showed that treatment with $2 \mu \mathrm{M}$ olaparib for $2 \mathrm{~h}$ markedly inhibited PARylation of cellular proteins in a monocyte preparation that presented high basal levels of PARP activity (Fig. 4B). The same olaparib concentration abrogated migration in response to PIGF of all monocyte preparations tested with similar efficacy compared to the anti-VEGFR-1 D16F7 mAb (Fig. 4C and D).

Influence of olaparib on PlGF-induced signaling pathways in monocytes. It has been demonstrated that PlGF acts as survival factor for tumor cells by upregulating nuclear factor $-\kappa \mathrm{B}(\mathrm{NF}-\kappa \mathrm{B})$ activity (24). Therefore, we investigated whether PlGF-mediated stimulation of monocytes might result in NF- $\kappa \mathrm{B}$ induction and whether olaparib modulates $\mathrm{NF}-\kappa \mathrm{B}$ 
A

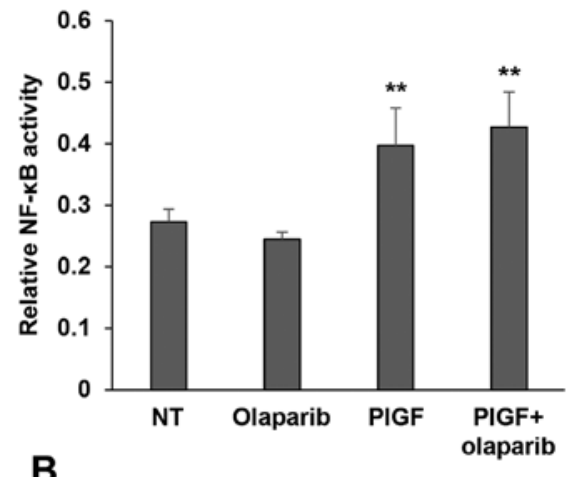

B

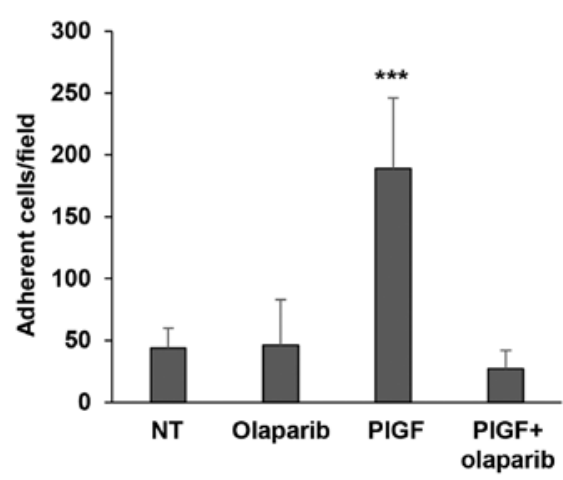

Figure 5. Olaparib inhibits PlGF-induced monocyte adhesion to fibronectin while it does not affect NF- $\mathrm{kB}$ activation by the angiogenic factor. (A) Influence of olaparib on NF-kB activation in response to PlGF. Monocytes, either vehicle (not treated, NT) or olaparib pre-treated ( $2 \mu \mathrm{M}$ for $2 \mathrm{~h}$ ), were incubated in the presence of $50 \mathrm{ng} / \mathrm{ml}$ PlGF for $20 \mathrm{~h}$. Whole-cell extracts were then analyzed for NF- $\mathrm{kB}$ activity by a quantitative Trans-AM kit, as described in Materials and methods. Data are representative of one out of three independent experiments with similar results and are the mean values from three independent determinations $\pm \mathrm{SD}$. Results of statistical analysis using one-way ANOVA followed by Bonferroni's post-test were as follows: ** $\mathrm{P}<0.01$, PlGF vs. NT, PlGF vs. olaparib; ${ }^{* *} \mathrm{P}<0.01, \mathrm{PlGF}+$ olaparib vs. NT and PlGF + olaparib vs. olaparib; differences between PlGF and PIGF + olaparib were not significant. (B) Influence of olaparib on monocyte adhesion to fibronectin. The ability of control or PlGF-stimulated monocytes $(100 \mathrm{ng} / \mathrm{ml})$ to adhere to fibronectin was evaluated, as described in Materials and methods, in vehicle (not treated, NT) or olaparib $(2 \mu \mathrm{M}$ for $2 \mathrm{~h}$ ) pre-treated cells. Histogram represents the mean values of the number of adherent cells/field \pm SD. Results of statistical analysis using one-way ANOVA followed by Bonferroni's post-test were as follows: ${ }^{* * * *} \mathrm{P}<0.001, \mathrm{PlGF}$ vs. NT, PlGF vs. olaparib and PlGF vs. PlGF + olaparib; differences between $\mathrm{NT}$, olaparib and PIGF+olaparib were not significant.

activation. Results indicated that PIGF caused a significant increase in NF- $\mathrm{\kappa B}$ activity that was not affected by pre-treatment with olaparib (Fig. 5A).

In order to shed light on the mechanism of the olaparibmediated inhibition of monocyte chemotaxis and invasiveness stimulated by PIGF and based on PARPi ability to modulate integrin expression in leukocytes (17), we evaluated the influence of pre-treatment with olaparib on monocyte adhesion to fibronectin, a process that requires integrin activation (25). Analysis of cell adhesion to fibronectin revealed that PIGF strongly induced monocytes to adhere to this ECM component and that olaparib hampered this effect (Fig. 5B). These data strongly suggested that inhibition of integrin activation by olaparib may contribute to the observed effects on the motility of PlGF-stimulated monocytes.

\section{Discussion}

In the present study, we demonstrated for the first time that the PARPi olaparib hampers PIGF-driven stimulation of myelomonocytic cells. This effect is due, at least in part, to inhibition of integrin activation that seems to be required for monocytic cell ability to migrate and invade the ECM in response to VEGFR-1 activation by PIGF. The inhibitory activity of olaparib on monocytic cells is indeed comparable to that exerted by the recently described anti-VEGFR-1 D16F7 mAb (11).

Besides its role as an angiogenic factor in tumor-associated vascularization, PIGF has been shown to influence the aggressiveness of tumor cells from different tissue origin. Actually, PlGF is upregulated in many human cancer types, where its expression directly correlates with tumor stage, metastasis or recurrence, and inversely correlates with survival $(24,26)$. In addition, PIGF plasma levels are frequently high in patients treated with anti-VEGF agents $(24,26)$, suggesting an involvement in innate and/or acquired resistance mechanisms to these therapies (27-29). Indeed, PIGF can directly affect tumor cells increasing or inducing migration and ECM invasion as demonstrated in colorectal, pancreatic or breast carcinomas and melanoma (11,30-32). Furthermore, the responsiveness to PIGF of cancer cell lines requires the expression of VEGFR-1 (33).

PIGF secreted by tumor cells also contributes to the recruitment of monocytes/macrophages into the tumor mass (i.e., TAMs) and is involved in TAM polarization to a pro-angiogenic/immune-suppressive M2-like phenotype (34). M2-like TAMs secrete a number of growth factors and proteases that promote angiogenesis and ECM remodeling, and suppress antitumor immune responses thereby stimulating tumor growth, invasion and metastasis (35).

In this context, the inhibitory effects of olaparib on monocytic cell activation by PIGF suggests a role for this PARPi in pathological states where PIGF is overexpressed. Due to their ability of inhibiting DNA repair, PARPi were initially developed as radio- and chemosensitizers and thereafter approved as a novel class of anticancer drugs to be used in monotherapy for homologous recombination defective tumors. Notably, these compounds have been recently proposed as potentially effective therapeutic agents for a variety of non-oncological disorders in which DNA-damage-dependent and -independent mechanisms of PARP activation may play a pathophysiological role (14). Therefore, olaparib treatment might be of benefit also for other non-cancerous diseases that are associated with monocyte activation in response to PlGF through inhibition of signal transduction mechanisms and independently on its effects on DNA repair.

Regarding the mechanisms by which olaparib modulates the monocytic cell response to PIGF, we found that the PARPi, at concentrations below the plasma peak values detected in treated cancer patients (36-38) and that totally abrogate cellular PARP activity, hampered PlGF-induced monocyte adhesion to fibronectin, while it did not affect NF- $\mathrm{KB}$ activation in response to this angiogenic factor.

The transcription factor NF- $\mathrm{KB}$ plays a key role in the regulation of cell proliferation, inflammation, angiogenesis and suppression of apoptosis, and, when constitutively activated, may be critical in the development of drug resistance in tumor cells (reviewed in ref. 39). PIGF significantly increases tumor 
cell resistance to chemotherapy and this effect is associated with activation of NF- $\mathrm{kB}$ signaling pathways (24). Our results showed that PlGF induces NF- $\kappa \mathrm{B}$ activation also in human monocytes. However, monocyte treatment with olaparib did not prevent PlGF-induced NF- $\mathrm{BB}$ upregulation, suggesting that the inhibitory effect of olaparib on chemotaxis might involve alternative mechanisms. Nevertheless, it cannot be excluded that olaparib might affect NF- $\mathrm{kB}$ translocation to the nucleus potentially promoted by PIGF and further studies are in progress to clarify this issue.

On the other hand, we found that PlGF markedly stimulated monocyte adhesion to the ECM component fibronectin, which suggests that integrin activation may be crucial for the promotion of growth factor-induced cell motility. Adhesion of monocytes to ECM (through fibronectin) or to activated endothelial cells (through the adhesion molecule VCAM-1) is regulated by integrin $\beta 1$ conformational changes (40). In fact, integrin must switch from an inactive (closed/non-adherent) to an active (open/adherent) form. This inside-out change exposes the integrin binding site and is regulated by stimulation of $\mathrm{G}$ protein coupled receptors via intracellular signals (40). Notably, PI3K has a crucial role in the regulation of monocyte integrin activation (41) and migration (42). Indeed, PlGF-mediated stimulation of VEGFR-1 in primary monocytes results in the phosphorylation of PI3K, ERK1/2, p38 and Akt (Ser473) kinases, PI3K being a central regulator of PlGF-induced human monocytes chemotaxis (42). To this regard, it has been reported that several PARPi down-modulate monocyte adhesion to microvascular endothelial cells by preventing the conformational activation of integrin $\beta 1$ (16), which is required for inflammatory cell mobility. Consistently, we observed that olaparib pre-treatment abrogated the stimulating effect of PlGF on monocytic cell adhesion to fibronectin. Although results on olaparib ability to inhibit chemotaxis and ECM invasion activity were obtained using several human monocyte preparations, only one myelomonocytic cell line was tested in the present study. A greater number of cell lines would be required to strengthen our conclusion.

It could be, therefore, hypothesized that activation of VEGFR-1 by PIGF in monocytic cells might trigger signal transduction pathways that result in two different effects: proliferation, suppression of apoptosis and survival, which depend on NF- $\kappa \mathrm{B}$ activity; cell adhesion and migration, which depend on integrin activation. Our results using the PARPi olaparib are in agreement with this hypothesis. Actually, the data herein described suggest that olaparib interferes with a specific signaling pathway triggered by PlGF through VEGFR-1, which involves specific integrin activation, and that inhibition of PlGF-induced monocyte activation may contribute to PARPi antitumor activity.

\section{Acknowledgements}

Not applicable.

\section{Funding}

The present study was supported by the Italian Association for Cancer Research (AIRC, Investigator Grants IG 2013 N. 14042 and IG 2017 N. 20353 to G.G.) and in part by the Italian Ministry of Health (RC2017 project no. 2630202 to P.M.L.).

\section{Availability of data and materials}

Non applicable.

\section{Authors' contributions}

PML and GG participated in the research design, performed data analysis and wrote the manuscript. MGA, FR and LT performed the experiments. The final version of the manuscript has been read and approved by all authors.

\section{Ethics approval and consent to participate}

In regard to buffy coat use, all healthy donors signed an informed consent.

\section{Consent for publication}

Non applicable.

\section{Competing interests}

The authors declare that they have no competing interests.

\section{References}

1. Clauss M, Weich H, Breier G, Knies U, Röckl W, Waltenberger J and Risau W: The vascular endothelial growth factor receptor Flt-1 mediates biological activities. Implications for a functional role of placenta growth factor in monocyte activation and chemotaxis. J Biol Chem 271: 17629-17634, 1996.

2. Kim KJ, Cho CS and Kim WU: Role of placenta growth factor in cancer and inflammation. Exp Mol Med 44: 10-19, 2012.

3. Ding Y, Huang Y, Song N, Gao X, Yuan S, Wang X, Cai H, Fu Y and Luo Y: NFAT1 mediates placental growth factor-induced myelomonocytic cell recruitment via the induction of TNF-alpha. J Immunol 184: 2593-2601, 2010.

4. Kerber M, Reiss Y, Wickersheim A, Jugold M, Kiessling F, Heil M, Tchaikovski V, Waltenberger J, Shibuya M, Plate KH and Machein MR: Flt-1 signaling in macrophages promotes glioma growth in vivo. Cancer Res 68: 7342-7351, 2008.

5. Laurent J, Hull EF, Touvrey C, Kuonen F, Lan Q, Lorusso G, Doucey MA, Ciarloni L, Imaizumi N, Alghisi GC, et al: Proangiogenic factor PIGF programs CD $11 b^{+}$myelomonocytes in breast cancer during differentiation of their hematopoietic progenitors. Cancer Res 71: 3781-3791, 2011.

6. Czepluch FS, Olieslagers S, van Hulten R, Vöö SA and Waltenberger J: VEGF-A-induced chemotaxis of CD16+ monocytes is decreased secondary to lower VEGFR-1 expression. Atherosclerosis 215: 331-338, 2011.

7. Lin YL, Liang YC and Chiang BL: Placental growth factor down-regulates type $1 \mathrm{~T}$ helper immune response by modulating the function of dendritic cells. J Leukoc Biol 82: 1473-1480, 2007.

8. Zhou X and Qi Y: Larynx carcinoma regulates tumor-associated macrophages through PLGF signaling. Sci Rep 5: 10071, 2015.

9. Selvaraj SK, Giri RK, Perelman N, Johnson C, Malik P and Kalra VK: Mechanism of monocyte activation and expression of proinflammatory cytochemokines by placenta growth factor. Blood 102: 1515-1524, 2003.

10. Pagani E, Ruffini F, Antonini Cappellini GC, Scoppola A, Fortes C, Marchetti P, Graziani G, D'Atri S and Lacal PM: Placenta growth factor and neuropilin-1 collaborate in promoting melanoma aggressiveness. Int J Oncol 48: 1581-1589, 2016.

11. Graziani G, Ruffini F, Tentori L, Scimeca M, Dorio AS, Atzori MG, Failla CM, Morea V, Bonanno E, D'Atri S and Lacal PM: Antitumor activity of a novel anti-vascular endothelial growth factor receptor-1 monoclonal antibody that does not interfere with ligand binding. Oncotarget 7: 72868-72885, 2016. 
12. Atzori MG, Tentori L, Ruffini F, Ceci C, Lisi L, Bonanno E, Scimeca M, Eskilsson E, Daubon T, Miletic H, et al: The anti-vascular endothelial growth factor receptor-1 monoclonal antibody D16F7 inhibits invasiveness of human glioblastoma and glioblastoma stem cells. J Exp Clin Cancer Res 36: 106, 2017.

13. Atzori MG, Tentori L, Ruffini F, Ceci C, Bonanno E, Scimeca M, Lacal PM and Graziani G: The anti-vascular endothelial growth factor receptor-1 monoclonal antibody D16F7 inhibits glioma growth and angiogenesis in vivo. J Pharmacol Exp Ther 364 77-86, 2018.

14. Berger NA, Besson VC, Boulares AH, Bürkle A, Chiarugi A, Clark RS, Curtin NJ, Cuzzocrea S, Dawson TM, Dawson VL, et al: Opportunities for the repurposing of PARP inhibitors for the therapy of non-oncological diseases. Br J Pharmacol 175: 192-222, 2018

15. Martin-Hernandez K, Rodriguez-Vargas JM, Schreiber V and Dantzer F: Expanding functions of ADP-ribosylation in the maintenance of genome integrity. Semin Cell Dev Biol 63 : 92-101, 2017.

16. Bai $\mathrm{P}$ and Virág L: Role of poly(ADP-ribose) polymerases in the regulation of inflammatory processes. FEBS Lett 586: 3771-3777, 2012.

17. Rom S, Zuluaga-Ramirez V, Reichenbach NL, Dykstra H, Gajghate S, Pacher P and Persidsky Y: PARP inhibition in leukocytes diminishes inflammation via effects on integrins/cytoskeleton and protects the blood-brain barrier. J Neuroinflammation 13: 254, 2016

18. Shah GM, Robu M, Purohit NK, Rajawat J, Tentori L and Graziani G: PARP inhibitors in cancer therapy: Magic bullets but moving targets. Front Oncol 3: 279, 2013.

19. Pujade-Lauraine E, Ledermann JA, Selle F, Gebski V, Penson RT, Oza AM, Korach J, Huzarski T, Poveda A, Pignata S, et al: Olaparib tablets as maintenance therapy in patients with platinum-sensitive, relapsed ovarian cancer and a $B R C A 1 / 2$ mutation (SOLO2/ENGOT-Ov21): A double-blind, randomised, placebo-controlled, phase 3 trial. Lancet Oncol 18: 1274-1284, 2017.

20. Robson M, Im SA, Senkus E, Xu B, Domchek SM, Masuda N, Delaloge S, Li W, Tung N, Armstrong A, et al: Olaparib for metastatic breast cancer in patients with a Germline BRCA mutation. N Engl J Med 377: 523-533, 2017.

21. Faraoni I, Compagnone M, Lavorgna S, Angelini DF, Cencioni MT, Piras E, Panetta P, Ottone T, Dolci S, Venditti A, et al: BRCA1, PARP1 and $\gamma \mathrm{H} 2 \mathrm{AX}$ in acute myeloid leukemia: Role as biomarkers of response to the PARP inhibitor olaparib. Biochim Biophys Acta 1852: 462-472, 2015.

22. Ruffini F, Failla CM, Orecchia A, Bani MR, Dorio AS, Fortes C, Zambruno G, Graziani G, Giavazzi R, D'Atri S, et al: Expression of the soluble vascular endothelial growth factor receptor-1 in cutaneous melanoma: Role in tumour progression. $\mathrm{Br} \mathrm{J}$ Dermatol 164: 1061-1070, 2011.

23. Lacal PM, Morea V, Ruffini F, Orecchia A, Dorio AS, Failla CM Soro S, Tentori L, Zambruno G, Graziani G, et al: Inhibition of endothelial cell migration and angiogenesis by a vascular endothelial growth factor receptor-1 derived peptide. Eur J Cancer 44: 1914-1921, 2008

24. Levati L, Ruffini F, Muzi A, Umezawa K, Graziani G, D'Atri S and Lacal PM: Placenta growth factor induces melanoma resistance to temozolomide through a mechanism that involves the activation of the transcription factor NF- $\mathrm{BB}$. Int J Oncol 38: 241-247, 2011.

25. White ES, Livant DL, Markwart S and Arenberg DA: Monocyte-fibronectin interactions, via alpha(5)beta(1) integrin, induce expression of CXC chemokine-dependent angiogenic activity. J Immunol 167: 5362-5366, 2001.
26. Dewerchin $\mathrm{M}$ and Carmeliet P: Placental growth factor in cancer. Expert Opin Ther Targets 18: 1339-1354, 2014.

27. Jain RK, Duda DG, Willett CG, Sahani DV, Zhu AX, Loeffler JS, Batchelor TT and Sorensen AG: Biomarkers of response and resistance to antiangiogenic therapy. Nat Rev Clin Oncol 6: 327-338, 2009.

28. Bagley RG, Ren Y, Weber W, Yao M, Kurtzberg L, Pinckney J, Bangari D, Nguyen C, Brondyk W, Kaplan J and Teicher BA: Placental growth factor upregulation is a host response to antiangiogenic therapy. Clin Cancer Res 17: 976-988, 2011.

29. Carmeliet $P$ and Jain RK: Molecular mechanisms and clinical applications of angiogenesis. Nature 473: 298-307, 2011.

30. FischerC,Jonckx B,Mazzone M,ZacchignaS,Loges S, PattariniL, Chorianopoulos E, Liesenborghs L, Koch M, De Mol M, et al: Anti-PlGF inhibits growth of $\operatorname{VEGF(R)-inhibitor-resistant~}$ tumors without affecting healthy vessels. Cell 131: 463-475, 2007.

31. Coenegrachts L, Maes C, Torrekens S, Van Looveren R, Mazzone M, Guise TA, Bouillon R, Stassen JM, Carmeliet P and Carmeliet G: Anti-placental growth factor reduces bone metastasis by blocking tumor cell engraftment and osteoclast differentiation. Cancer Res 70: 6537-6547, 2010.

32. Wei SC, Tsao PN, Weng MT, Cao Z and Wong JM: Flt-1 in colorectal cancer cells is required for the tumor invasive effect of placental growth factor through a p38-MMP9 pathway. J Biomed Sci 20: 39, 2013

33. Yao J, Wu X, Zhuang G, Kasman IM, Vogt T, Phan V, Shibuya M, Ferrara $\mathrm{N}$ and Bais C: Expression of a functional VEGFR-1 in tumor cells is a major determinant of anti-PIGF antibodies efficacy. Proc Natl Acad Sci USA 108: 11590-11595, 2011

34. Rolny C, Mazzone M, Tugues S, Laoui D, Johansson I, Coulon C, Squadrito ML, Segura I, Li X, Knevels E, et al: HRG inhibits tumor growth and metastasis by inducing macrophage polarization and vessel normalization through downregulation of PlGF. Cancer Cell 19: 31-44, 2011.

35. Galdiero MR, Garlanda C, Jaillon S, Marone G and Mantovani A: Tumor associated macrophages and neutrophils in tumor progression. J Cell Physiol 228: 1404-1412, 2013.

36. Fong PC, Boss DS, Yap TA, Tutt A, Wu P, Mergui-Roelvink M, Mortimer P, Swaisland H, Lau A, O'Connor MJ, et al: Inhibition of poly(ADP-ribose) polymerase in tumors from $B R C A$ mutation carriers. N Engl J Med 361: 123-134, 2009.

37. Roth J, Peer CJ, Mannargudi B, Swaisland H, Lee JM, Kohn EC and Figg WD: A sensitive and robust ultra HPLC assay with tandem mass spectrometric detection for the quantitation of the PARP inhibitor olaparib (AZD2281) in Human plasma for pharmacokinetic application. Chromatography 1: 82-95, 2014.

38. Plummer R, Swaisland H,Leunen K, van Herpen CM, Jerusalem G, De Grève J, Lolkema MP, Soetekouw P, Mau-Sørensen M, Nielsen D, et al: Olaparib tablet formulation: Effect of food on the pharmacokinetics after oral dosing in patients with advanced solid tumours. Cancer Chemother Pharmacol 76: 723-729, 2015.

39. Xia Y, Shen S and Verma IM: NF- $\kappa$ B, an active player in human cancers. Cancer Immunol Res 2: 823-830, 2014.

40. Askari JA, Buckley PA, Mould AP and Humphries MJ: Linking integrin conformation to function. J Cell Sci 122: 165-170, 2009.

41. Ferreira AM, Isaacs H, Hayflick JS, Rogers KA and Sandig M The p110delta isoform of PI3K differentially regulates betal and beta 2 integrin-mediated monocyte adhesion and spreading and modulates diapedesis. Microcirculation 13: 439-456, 2006.

42. Tchaikovski V, Fellbrich G and Waltenberger J: The molecular basis of VEGFR-1 signal transduction pathways in primary human monocytes. Arterioscler Thromb Vasc Biol 28: 322-328, 2008. 